\title{
Analytical quantification of the inflammatory cell infiltrate and CD95R expression during treatment of drug-induced toxic epidermal necrolysis
}

Received: 15 December 2004 / Revised: 8 June 2005/ Accepted: 23 July 2005 / Published online: 25 October 2005

(C) Springer-Verlag 2005

\begin{abstract}
The treatment of drug-induced toxic epidermal necrolysis (TEN) remains unsatisfactory. Intravenous immunoglobulins (IVIg) and intravenous cyclosporin A (CsA) have shown some efficacy in short series of patients. We assessed the effects of IVIg and CsA on TEN lesional and apparently uninvolved skin using standard histology and immunohistochemistry. Cutaneous biopsies were taken from necrotic and clinically uninvolved TEN skin at admission (D1) before any treatment, and after a 5-day treatment (D5). Two IVIg-treated patients $(0.75 \mathrm{~g} / \mathrm{kg} / \mathrm{day})$, two CsA-treated patients $(5 \mathrm{mg} / \mathrm{kg} / \mathrm{day})$ and two control patients only receiving supportive care were compared. Biopsies were examined by standard histology and immunohistochemistry using antibodies directed to CD68 antigen (macrophages), CD45R0 antigen (activated T lymphocytes), Factor XIIIa (dermal dendrocytes) and the CD95 receptor (apoptosis marker). The different cell densities were evaluated by computerized image analysis. The clinical outcomes with the different treatments were also recorded. There was no obvious difference in the duration of hospitalization in intensive care unit between the three groups but one patient passed away in each of the IVIg- and CsAgroups. At D5, no differences were found between the three groups in the histological and clinical rate of reepithelialization, and in the evolution of T lymphocyte, macrophage and dendrocyte densities in the epidermis and dermis. However, the expression of the CD95
\end{abstract}

P. Paquet $(\bowtie) \cdot$ G. Piérard

Department of Dermatopathology, University Hospital of Liège, CHU Sart Tilman, 4000 Liege, Belgium

E-mail: P.Paquet@chu.ulg.ac.be

Tel.: + 32-4-3662408

Fax: + 32-4-3662976

E. Jacob $\cdot$ P. Damas

Intensive care medicine, burn center, University Hospital of Liège, 4000 Liege, Belgium

J. Pirson

Intensive care medicine, burn center, Brussels Military Hospital, 1120 Brussels, Belgium receptor was similarly and strongly abated at D5 in the epidermis of IVIg- and CsA-treated patients, while it was conversely increased in the two patients under supportive care only. Such a difference was found both in necrotic and uninvolved sites. IVIg and CsA treatments thus appeared to exert no obvious effect on the inflammatory infiltrate, but both abated the expression of the CD95 receptor in the skin of TEN patients. This effect did not seem sufficient to fully reverse the clinical evolution of the disease. It is inferred that IVIg and CsA do not completely abate the TEN process.

Keywords Toxic epidermal necrolysis - Cyclosporin A . Immunoglobulin

Drug-induced toxic epidermal necrolysis (TEN) is a lifethreatening disease characterized by extensive destruction of the epidermis [5]. To date, no cause other than drugs has been found in TEN although $5 \%$ of the cases remain "idiopathic" [22]. The mortality rate, which is higher than $40 \%$ in patients with more than $30 \%$ skin detachment is mainly due to septicemia and metabolic disturbances following the loss of epidermal integrity [11].

The mechanism leading to massive keratinocyte death in TEN is thought to be apoptosis [18]. Disregulations in the tumor necrosis factor- $\alpha(\mathrm{TNF}-\alpha)$ pathway, CD95 system (Fas ligand-CD95L, Fas receptor-CD95R) and calcium homeostasis in the epidermis are likely involved [18]. Indeed, TEN keratinocytes express large amounts of active CD95L able to induce apoptosis of CD95R-positive cells. CD95R, which belongs to the $\mathrm{TNF} / \mathrm{NGF}$ receptor family is over-expressed in TEN epidermis.

The TEN initiation phase leading to keratinocyte apoptosis is followed by an amplification phase involving inflammatory cells [18]. An active role has been ascribed to $\mathrm{T}$ lymphocytes, particularly CD4 + cells in the dermis and CD $8+$ cells in the epidermis. In addition, 
L1-protein-enriched macrophages and Factor XIIIapositive dendrocytes appear also to play pathogenic roles in the epidermis and dermis, respectively.

In the past, systemic corticosteroids were used for targeting the inflammatory reaction in TEN [8]. However, there was no evidence for a benefit in the healing process, while they worsened the prognosis by increasing the risk of septicemia [8]. Only a few case reports have reported other treatment modalities including cyclophosphamide [6], pentoxyfilline [23], thalidomide [31] and cyclosporin A (CsA) [1, 9, 12, 15, 18, 21, 25, 26, 32]. Although T lymphocytes are the main target of CsA, the drug could also somewhat prevent keratinocyte apoptosis in TEN [15]. Some TEN patients have also been treated with intravenous human immunoglobulins (IVIg) $[2,10,14,19,20,24,28-30]$. The rationale for this treatment was to block CD95R on keratinocytes. The early promising clinical results of IVIg treatment in TEN were subsequently challenged [2]. Histological and immunohistological assessments of the IVIg effects in TEN skin have not been studied so far.

We presently report on the modifications in the inflammatory infiltrate ( $\mathrm{T}$ lymphocytes, macrophages, type I dendrocytes) and in the epidermal CD95R expression during IVIg and CsA treatments. Both of these treatments were administered to two TEN patients. A comparison was made with supportive care given to two other TEN patients.

TEN was diagnosed in six patients admitted to the burn units of the University Hospital of Liège and the Brussels Military Hospital (Belgium). The diagnosis was established following previously defined criteria [4]. In each case, a recent drug intake was disclosed. The salient characteristics of the patients are listed in Table 2. The disease duration on admission was recorded since the first day of erythematous rash or mucous membrane involvement. Our center representing a secondary reference center, the disease duration on admission was sometimes quite long. On admission, the patients were placed on a fluidized bed and benefited from supportive and antiseptic care measures including daily baths. In two randomized patients, IVIg (Sandogobuline ${ }^{\circledR}$, Novartis Pharma, Basel, Switzerland) was administered daily at a dose of $0.75 \mathrm{~g} / \mathrm{kg}$ body weight for five consecutive days. These IVIg batches were not tested for their antiFas activity. In two other randomized patients, intravenous CsA (Neoral Sandimmun ${ }^{\circledR}$, Novartis Pharma, Basel, Switzerland) was administered at a daily dosage of $5 \mathrm{mg} / \mathrm{kg}$ body weight for five consecutive days. We retrieved from our files skin biopsies from two other patients who had been hospitalized in the mid-nineties, at a time when IVIg and CsA were not advocated in TEN. These patients had received supportive care only, without any drug currently considered active in TEN.

Punch biopsies were performed at admission (D1). They were taken from early bullous lesions $(<24 \mathrm{~h})$, and from the apparently uninvolved skin at $2 \mathrm{~cm}$ outside a blister. They were formalin-fixed and paraffin-embedded. Five days later (D5), two other biopsies were obtained next to the first samples, that is, one from an apparently intact skin area and the other from a previously altered area.

Slides were stained with hematoxylin and eosin. In addition, the three-step avidin-biotin method was applied to a panel of antibodies (Table 1). Appropriate positive controls (graft versus host reaction, bullous pemphigoid, staphylococcal scalded skin syndrome) and negative controls (normal skin) were considered [16].

Computerized image analysis (Leica imaging systems, Qwin, Cambridge, UK) was used to assess the cell densities relative to each immunolabeling. Blinded evaluations were performed by an observer unaware of the clinical condition and treatment. Five contiguous representative fields $(\times 400)$ located in the central part of the damaged epidermis were analyzed in each lesion. The cell densities were expressed as the number of positive cells per surface unit $\left(10,000 \mu \mathrm{m}^{2}\right)$. The antiCD95R antibody labeled keratinocytes as well as some inflammatory cells in the epidermis. Image analysis allowed to discriminate among these cell types by only recording the largest positive cells corresponding to the keratinocytes.

Re-epithelialization at D5 appeared to be moderately improved in IVIg and CsA-treated patients compared to the untreated patients (Table 2). No difference was suggested between CsA- and IVIg-treated patients. However, the small number of patients does not permit firm conclusion in terms of re-epithelialization. No difference in time before hospital discharge was evidenced between the IVIg- and CsA-treated patients, and the untreated patients (Table 2). One patient of each of the IVIg-and CsA-groups died from sepsis.

At D1, subepidermal blisters resulted from complete epidermal necrosis underlined by a discrete mononuclear cell infiltrate. The clinically uninvolved skin looked histologically normal. At D5, the aspect of bullous skin remained similar in three patients (pts 2, 4, 5). A regenerative epidermis was present in three other patients (pts 1, 3, 6) without any residual blister. In these latter patients, a discrete superficial perivascular lymphoid infiltrate underlined the thickened epidermis. In all six patients, the clinically unaltered skin at D5
Table 1 Panel of monoclonal (Mo) and polyclonal (Po) antibodies

\begin{tabular}{llll}
\hline Antibody & Source & Dilution & Specificity \\
\hline Mo, UCLH1 anti-CD45R0 & Dako & $1: 100$ & Mature T cells \\
Po, anti-factor XIIIa & BioGenex & $1: 300$ & Type I dendrocytes \\
Mo, anti-CD95 (Fas) & Dako & $1: 25$ & CD95 receptor \\
Mo, anti-CD68 & Dako & $1: 50$ & Lysosomes in reactive \\
& & & macrophages \\
\hline
\end{tabular}




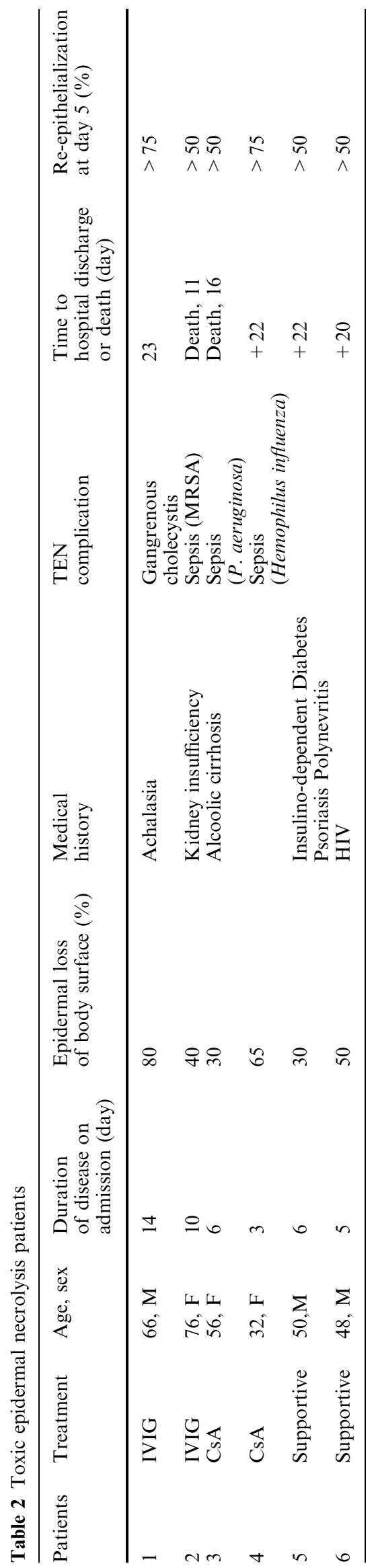

remained histologically normal or showed a discrete superficial perivascular lymphoid infiltrate.

Immunohistochemical results are presented in Tables 3 and 4. In bullous skin, Factor XIIIa + dendrocytes represented the most abundant cell population in the superficial dermis. Compared to the D1 pretreatment status, their number was reduced or remained similar at D5 in all patients but in patient 6 they were more numerous. At D1, few CD45R0 + T lymphocytes and rare CD68 + macrophages were disclosed in the epidermis and superficial dermis of all patients. These cell densities decreased or remained similar at D5. The most prominent difference between treated TEN patients and those under supportive care only was found in the CD95R + keratinocytes. Their number was greatly decreased at D5 in IVIg $(-95.8 \%$ and $-32.5 \%)$ and CsA-treated patients $(-37.9 \%$ and $-67.7 \%)$. In contrast, they markedly increased $(+450 \%$ and $+92.5 \%)$ in the two patients under supported care (Fig. 1). In the clinically uninvolved skin, Factor XIIIa + dendrocytes, CD45R0 + lymphocytes and CD68 + macrophages were in a small number in all TEN patients, without any obvious change in their densities between D1 and D5. CD95R was expressed on many keratinocytes at D1 in most of the biopsy specimens. In contrast, this immunoreactivity was disclosed in less than $1 \%$ of the keratinocytes in other non-TEN diseases. At D5, the number of CD95R + keratinocytes moderately increased in IVIg-treated TEN patients, decreased in CsA-treated patients and greatly increased in TEN patients under supportive care only (Fig. 2). In some TEN patients, the number of $\mathrm{CD} 95+$ keratinocytes was greater in the clinically uninvolved skin than in the corresponding bullous lesions, both at D1 and D5. However, due to the small number of TEN patients, no firm relationship was found between the clinical course and the densities of CD95R + keratinocytes and inflammatory cells.

High IVIg dosages were recently used to treat some TEN patients $[2,10,14,19,20,24,28-30]$. The rationale for this treatment relied on the in vitro inhibition of FasRFasL interaction and keratinocytes apoptosis by Ig. This effect is likely mediated by an anti-CD95R activity rather than by an interaction with CD95L. In vitro studies have also shown that high dose Ig inhibit CD95R-mediated apoptosis of keratinocyte in a dose-dependent relationship [27, 30]. However, the in vitro protective effect is observed at variable concentrations according to the different IVIg batches available for in vivo usage [20, 27]. This observation points to the difficulties in standardizing IVIg treatment of TEN. Preliminary clinical results about this treatment remain controversial so far, showing either improvement [10,14, 19, 20, 24, 28-30] or worsening [2] in TEN morbidity and mortality. All the clinical non comparative studies reported so far were not supported by histological examination.

Cyclosporin A was also proposed for reversing TEN progression [1, 9, 12, 15, 21, 25, 26, 32]. The suppressive action of CsA primarily targets T-cell functions, and it inhibits the macrophage activation [15]. CsA also re- 

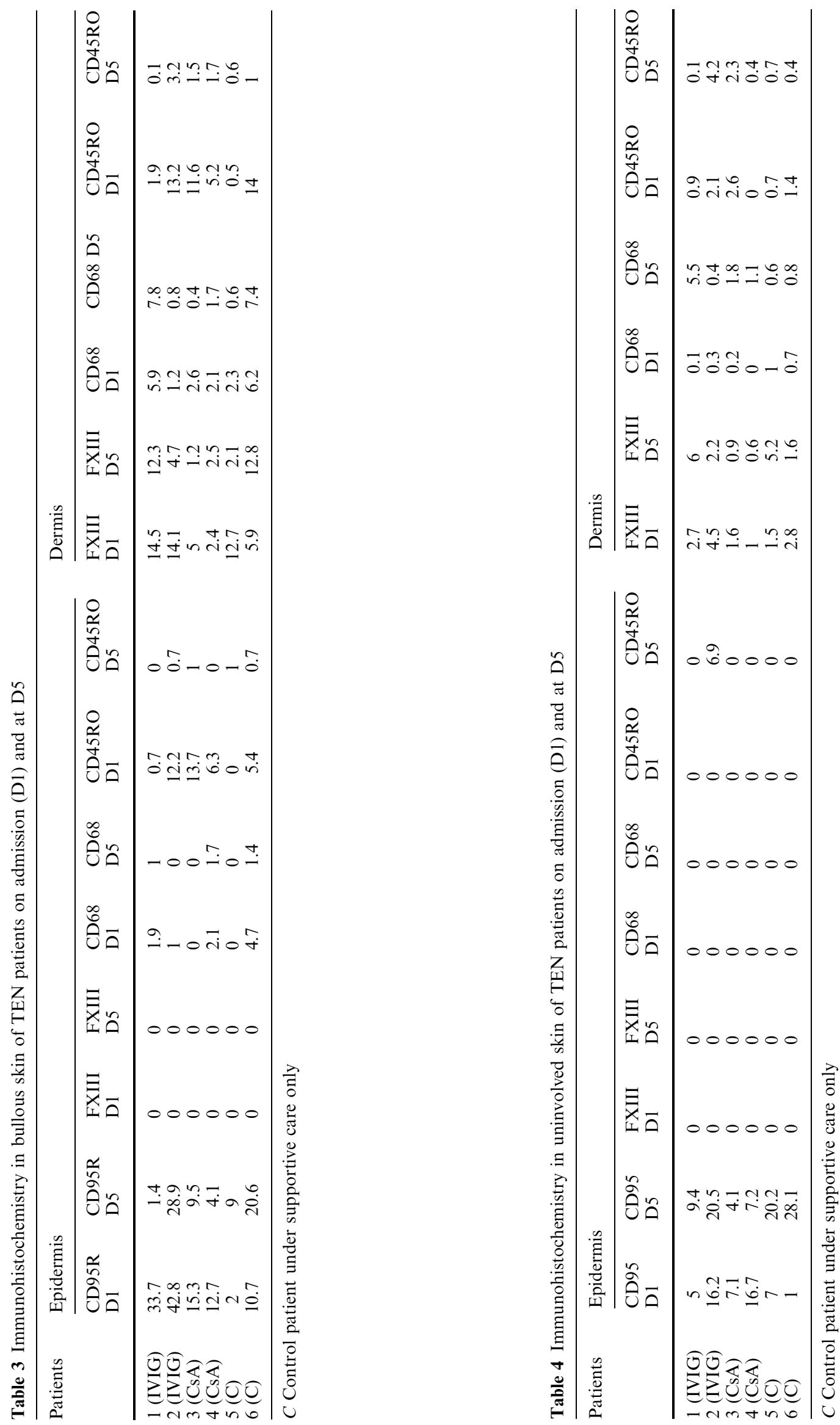
Fig. 1 Computerized image analysis of the expression of CD95R in necrotic TEN epidermis, before treatment (DI) and after a 5-day treatment with IVIG $(a 1, a 2)$ or CsA $(b 1, b 2)$ in comparison with a patient benefiting from supportive measures only $(c 1$, c2). CD95 + keratinocytes appear in blue
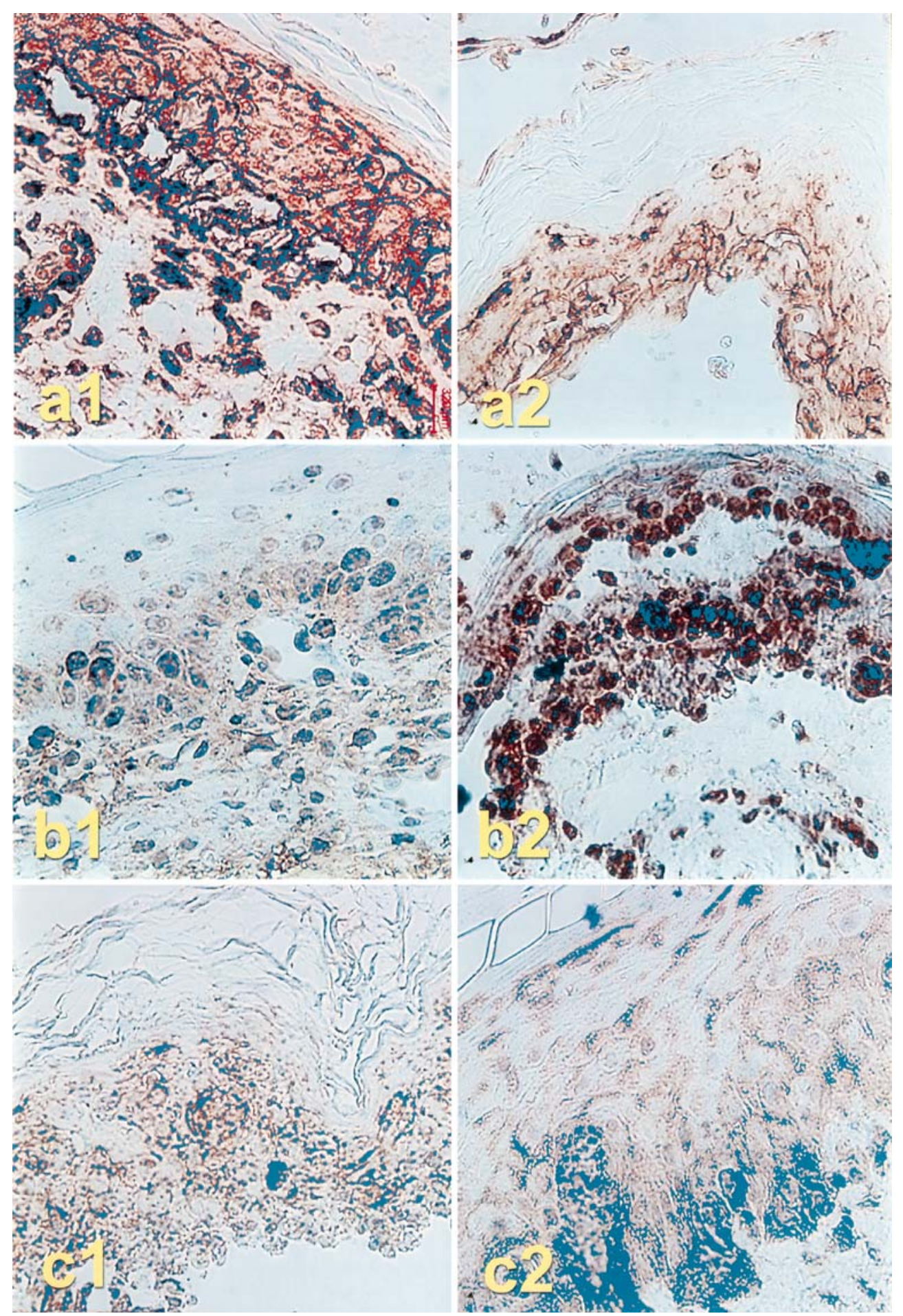

duces TNF- $\alpha$ secretion by human epidermal keratinocytes in vitro [15]. This cytokine is one of the major ones involved in TEN epidermal destruction. Treatment of mice with subcutaneously injected CsA was shown to inhibit TNF- $\alpha$ gene expression by about $70 \%$ in the skin [15]. In addition, the CsA immunosuppressive effect may partly be mediated by inhibition of the activation of the FasR-FasL system. In vitro, CsA inhibits the drug-induced expression of CD95R and CD95L-mRNA and blocks drug-mediated apoptosis of some cell types [7]. Only few TEN cases have been treated with CsA in uncontrolled studies [1, 9, 12, 21, 25, 26, 32]. Clinical beneficial effects were generally reported, but not supported by specific biological evaluations.

The apoptosis marker CD95R is shown here to be downregulated in clinically involved TEN skin at completion of a 5-day IVIg or CsA treatment. CD95R was moderately increased (IVIg) or decreased (CsA) in 
Fig. 2 Computerized image analysis of the expression of CD95R in clinically uninvolved TEN epidermis, before treatment $(D l)$ and after a 5day treatment with IVIG $(d 1$, $d 2)$ or CsA $(e 1, e 2)$ in comparison with a patient benefiting from supportive measures only $(f 1, f 2)$. CD95+ keratinocytes appear in blue
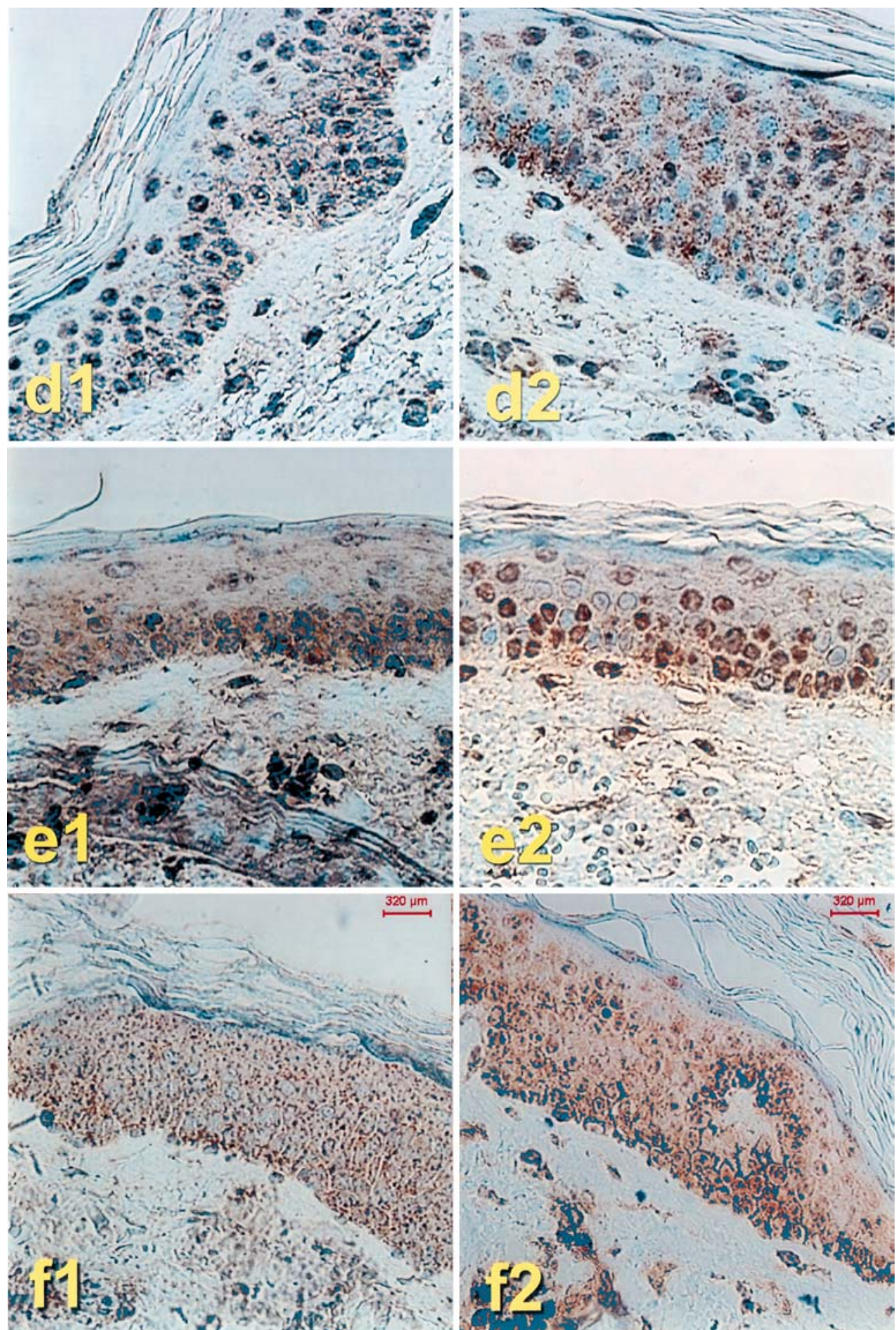

the clinically uninvolved skin of TEN patients. In contrast, in TEN patients under supportive care only, CD95R was markedly increased at D5 compared to D1 both in the uninvolved and involved skin. Hence, the present in vivo findings are in line with the in vitro inhibitory effects of IVIg and CsA on the CD95R-CD95L system. No effect of IVIg or CsA was evidenced on the densities of the TEN inflammatory cell infiltrate. This was surprising for CsA, which exhibits a potent inhibitory effect on activated $\mathrm{T}$ lymphocytes. However, the inability of CsA to downregulate some types of cutaneous cell inflammatory infiltrate was previously noticed, especially in alopecia areata [13].

Although both IVIg and CsA treatments inhibit the CD95R-CD95L system, this effect may not be powerful 
enough to initiate a clinically relevant improvement in re-epithelialization. Similarly, these drugs appear to fail to control TEN morbidity and mortality at least when major risk factors are present. In our series, one IVIgtreated patient died from sepsis. She was the oldest of the patients and she suffered from previous kidney deficiency. These factors are two negative predictive factors for TEN outcome [3]. The administered $0.75 \mathrm{~g} /$ $\mathrm{kg} /$ day IVIg dosage was also lower than the newly recommended dosage $(1 \mathrm{~g} / \mathrm{kg} /$ day $)$ [2, 20, 28]. Indeed, higher IVIg dosage might have impaired her renal function, leading to increased morbidity risk [2]. Sepsis was also the cause of death of one of the CsA-treated patients. This patient suffered from cirrhosis, which is a major mortality risk factor in TEN.

The densities of the inflammatory cell infiltrates were moderate in all TEN patients at D1. Only Factor $\mathrm{XIIIa}+$ dendrocytes appeared boosted at that time. This supports the concept considering that the early phase of the TEN process is probably initiated by the keratinocytes themselves and the dermal dendrocytes [18]. The pathomechanisms leading to keratinocyte destruction are probably multiple and different at each stage of epidermal maturation [17]. The apoptosis pattern may involve the calcium-independent CD95 system in the deeper epidermal layers and a calcium-dependent system in the superficial epidermis. The present findings suggest that the destruction of keratinocytes is provoked by simultaneous activation of several apoptosis pathways. Blocking CD95R may prevent the apoptosis progression, but has little effect on the epidermal healing phase. Indeed, the rate of re-epithelialization induced by both IVIg and CsA during the early TEN phase did not appear different from patients under supportive care only as assessed by the clinical outcome and by microscopy at D5. A regenerating epidermis was demonstrated in one CsA and one IVIg-treated patient, but also in one patient under supportive care only.

In conclusion, both IVIg $(0.75 \mathrm{~g} / \mathrm{kg} /$ day $)$ and CsA $(5 \mathrm{mg} / \mathrm{kg} /$ day) treatments appear to inhibit the apoptotic CD95 pathway in TEN patients. This effect might not be effective enough to improve the early clinical evolution and prognosis in TEN patients with high-risk factors.

Acknowledgements This work was supported by the "Fonds d'Investissement de la Recherche Scientifique" of the University Hospital of Liège

\section{References}

1. Arevalo J, Lorente JA, Gonzalez-Herrada C, Jimenez-Reyes J (2000) Treatment of toxic epidermal necrolysis with cyclosporin A. J Trauma Inj Inf Crit Care 48:473-478

2. Bachot N, Revuz J, Roujeau JC (2003) Intravenous immunoglobulin treatment for Stevens-Johnson syndrome and toxic epidermal necrolysis. Arch Dermatol 139:33-36

3. Bastuji-Garin S, Fouchard N, Bertocchi M, Roujeau JC, Revuz J, Wolkenstein P (2000) SCORTEN: a severity-of-illness score for toxic epidermal necrolysis. J Invest Dermatol 45:149-153
4. Bastuji-Garin S, Rzany B, Stern RS, Shear NH, Naldi L, Roujeau JC (1993) Clinical classification of cases of toxic epidermal necrolysis, Stevens-Johnson syndrome and erythema multiforme. Arch Dermatol 129:92-96

5. Becker DS (1998) Toxic epidermal necrolysis. Lancet 351:14171420

6. Frangogiannis NG, Boridy F, Mazhar M, Mathews R, Gangopadhyay S, Cate T (1996) Cyclophosphamide in the treatment of toxic epidermal necrolysis. South Med J 89:1001-1003

7. Fulda S, Sieverts H, Friesen C, Herr I, Debatin KM (1997) The CD95 (APO-1Fas) system mediates drug-induces apoptosis in neuroblastoma cells. Cancer Res 57:3823-3829

8. Herndon D (1995) Toxic epidermal necrolysis: a systemic and dermatologic disorder best treated with standard treatment protocols in burns intensive care units without the prolonged use of corticosteroids. J Am Coll Surg 180:340-342

9. Jarrett P, Rademaker M, Havill J, Pullon H (1997) Toxic epidermal necrolysis treated with cyclosporin and granulocyte colony simulating factor. Clin Exp Dermatol 22:146-147

10. Magina S, Lisboa C, Goncalves E, Conceicao P, Leal U, Mesquita-Guimaraes J (2000) A case of toxic epidermal necrolysis treated with intravenous immunoglobulin. Br J Dermatol 142:177-178

11. Mockenhaupt M, Norgauer J (2002) Cutaneous adverse drug reactions: Stevens-Johnson syndrome and toxic epidermal necrolysis. Allergy Clin Immunol Int 14:143-150

12. Murdoch SR, Fenton DA (1997) Toxic epidermal necrolysis and cyclosporin. Clin Exp Dermatol 22:250-256

13. Paquet P, Arrese Estrada J, Piérard GE (1992) Oral cyclosporin and alopecia aerata. Dermatology 105:314-315

14. Paquet P, Jacob E, Damas P, Piérard GE (2001) Treatment of drug-induced toxic epidermal necrolysis (Lyell's syndrome) with intravenous human immunoglobulins. Burns 27:652-655

15. Paquet P, Piérard GE (1999) Would cyclosporin A be beneficial to mitigate drug-induced toxic epidermal necrolysis? Dermatology 198:198-202

16. Paquet P, Piérard GE (2002) Differential patomechanisms of epidermal necrolytic blistering diseases. Int J Mol Med 10:695699

17. Paquet P, Piérard GE (2002) Keratinocyte injury in drug-induced toxic epidermal necrolysis: simultaneous but distinct topographic expression of CD95R and calprotectin. Int $\mathbf{J}$ Mol Med 10:145-147

18. Paquet P, Piérard GE, Quatresooz P (2005) Novel treatments for drug-induced toxic epidermal necrolysis (Lyell's syndrome). Int Arch Allergy Immunol 136:205-216

19. Phan TG, Wang R, Crotty T, Adelstein S (1999) Toxic epidermal necrolysis treated with intravenous gammaglobulin. Australas J Dermatol 40:153-157

20. Prins C, Kerdel FA, Padilla RS, Hunziker T, Chimenti S, Viard I et al (2003) Treatment of toxic epidermal necrolysis with highdose intravenous immunoglobulins. Arch Dermatol 139:26-32

21. Renfro L, Grant-Kels J, Daman LA (1989) Drug-induced toxic epidermal necrolysis treated with cyclosporin. Int $\mathbf{J}$ Dermatol 28:441-444

22. Roujeau JC, Guillaume JC, Fabre JP, Penso D, Flechet ML, Girre JP (1990) Toxic epidermal necrolysis (Lyell syndrome). Incidence and drug etiology in France, 1981-1985. Arch Dermatol 126:37-42

23. Sanclemente G, De la Roche C, Escobar C, Fabella R (1998) Pentoxifylline in toxic epidermal necrolysis and Stevens-Johnson syndrome. Int J Dermatol 38:873-874

24. Stella M, Cassano P, Bollero D, Clemente A, Giorio G (2001) Toxic epidermal necrolysis treated with intravenous high-dose immunoglobulins: our experience. Dermatology 203:45-49

25. Sullivan JR, Watson A (1996) Lamotrigine-induced toxic epidermal necrolysis treated with intravenous cyclosporin: a discussion of pathogenesis and immunosuppressive management. Australas J Dermatol 37:208-212

26. Szepietowki J, Wasik F, Szybejko-Machay G, May J (1997) Toxic epidermal necrolysis successfully treated with cyclosporin. Report of three cases. J Eur Acad Dermatol Venereol 9:169-172 
27. Trautmann A, Akdis M, Schmid-Grendelmeier P, Disch R, Bröcker EB, Blaser K et al (2001) Targeting keratinocyte apoptosis in the treatment of atopic dermatitis and allergic contact dermatitis. J Allergy Clin Immunol 108:839-846

28. Trent JT, Kirsner RS, Romanelli P, Kerdel FA (2003) Analysis of intravenous immunoglobulin for the treatment of toxic epidermal necrolysis using SCORTEN. Arch Dermatol 139:39-43

29. Tristani-Firouzi P, Petersen M, Saffle JR, Morris SE, Zone JJ (2002) Treatment of toxic epidermal necrolysis with intravenous immunoglobulin in children. $\mathbf{J}$ Am Acad Dermatol $47: 548-552$
30. Viard I, Wehrli P, Bullani R, Schneider P, Holler N, Salomon $D$ et al (1998) Inhibition of toxic epidermal necrolysis by blockade of CD95 with human intravenous immunoglobulin. Science 282:490-493

31. Wolkenstein P, Latarjet P, Roujeau JC (1998) Randomised comparison of thalidomide versus placebo in toxic epidermal necrolysis. Lancet 352:1586-1589

32. Zaki I, Patel S, Reed R, Dalziel KL (1995) Toxic epidermal necrolysis associated with severe hypocalcaemia, and treated with cyclosporin. Br J Dermatol 133:337-338 